\title{
Novel approach in iris shade matching: mobile photography and custom ocular shade guide
}

\author{
1Joseph Ginting, ${ }^{2}$ Haslinda Z Tamin, ${ }^{2}$ Putri Welda Utami Ritonga \\ ${ }^{1}$ Postgraduate Program in Prosthodontics \\ 2Department of Prosthodontics \\ Faculty of Dentistry, Universitas Sumatera Utara \\ Medan, Indonesia \\ Corresponding author: Joseph Ginting, E-mail: jdhg.prosth@gmail.com
}

\begin{abstract}
Introduction: Custom ocular prosthesis have several advantages such as even distribution of pressure on the eye socket, highly esthetic iris, better comfort and eyelid movements, but this prosthesis requires a long fabrication time. The fabrication step that spent time the most is the iris shade matching. Case: A 19 years-old woman with a defect in her left eye reported to Dental Hospital Universitas Sumatera Utara. The patient revealed history of infection of the left eye due to measles leading to surgical enucleation when she was one year old. Clinical examination shows healthy conjuctiva with no sign of infection or inflammation covering the posterior wall of the anopthalmic socket. Management: The iris shade matching was done using mobile photography and custom ocular shade guide, allowing the fabrication of iris button in the same day with shade matching appointment. The mobile photography eliminated the need for the operator painting skill and save the fabrication time significantly. The customoccular shade guide compensated the color biased during the photo taking procedure. Conclusion: The novel approach using mobile photography and custom ocular shade guide simplified the iris shade matching, provide highly esthetic results, eliminate the needs for operator painting skills, and save a significant amount of time in fabrication.
\end{abstract}

Keywords: custom ocular prosthesis, custom ocular shade guide, enucleation, anopthalmic.

This title has been presented in The 12th Biennial Congress of Asian Academy of Prosthodontics, 21 August 2021

\section{INTRODUCTION}

Loss of an eye can cause a significant psychological and emotional disturbance to any patient, because eyes are generally the first features of the face to be noted. ${ }^{1}$ Congenital defect, pathology or accidental trauma to eyes can lead to surgical intervention resulting in the removal of the eyeball. The surgical managementranges from evisceration, enucleation to exenteration depending upon the case and its severity. Evisceration is the removal of the contents of the globe while leaving the sclera and extraocular muscles intact. Enucleation is the removal of the eye from the orbit while preserving all other orbital structures. Exenteration is the mostradical of the three procedures and it involves removal of the eye and part of the bony orbit. ${ }^{1,2}$

The disfigurement caused by the loss of an eye can be treated with an ocular prosthesis. An ocular prosthesis which restores and replaces the natural eye aims to improve the patient's esthetics, restore and maintain the health of the remaining structures and consequently provide physical and mental well-being for the patient. ${ }^{2}$ There are two types of ocular prosthesis; stock ocular prosthesis (ready-made) or custom ocular prosthesis. Stock ocular prosthesis possess disadvantages such as ill-fitting and impropershade matching with the natural eyes, meanwhile the custom ocular prosthe- sis increases the adaptiveness, better estheticand movement of the eye ball, and exactly matches the iris position as that of the adjacent natural eye, but it requires more time in the fabrication process. ${ }^{3}$

The mostconsuming time in the ocular prosthesis fabrication is in the iris shade matching process. The conventional methods using paint to recreate the patient iris, depends on the clinician painting skills to produce an esthetic iris, it also requires days of waiting for the paint to dry before we can use it to make iris button or to attach it to the acrylic sclera. Nowadays, mobile photography grows rapidy in terms of sensorquality, resolution and lens sophistication. Some new mobile phone cameras have dual and even triple cameras setups including macrolenses which allow the smart phone to take picture of small object with great details. The smartphone camera is also lightweight, low cost and easy to operate. ${ }^{4}$ Using the rightmethods mobile photography can be used to recreate the patient iris with great details in shortamount of time, decreasing the time and visits that require in the custom ocular prosthesis fabrication significantly. ${ }^{4}$ This case report describe anovel approach in iris shade matching using mobile photography and a custom ocular shade guide.

\section{CASE}

A 19years-old woman with a defect in the left 
eye reported to Dental Hospital Universitas Sumatera Utara. The patient revealed a history of infection of the left eye due to measles leading to surgical enucleation when she was one year old. Clinical examination shows healthy conjuctiva with no sign of infection or inflammation covering the posterior wall of the anopthalmic socket. According to the treatment-based classification system given by Himanshi et al., the patient was categorized under Class 4 phthisis bulbi (severe enophthalmos with disfigured sclera and loss of orbital fat). ${ }^{5}$ This case (Fig. 1 ) was managed using a custom ocular prosthesis.

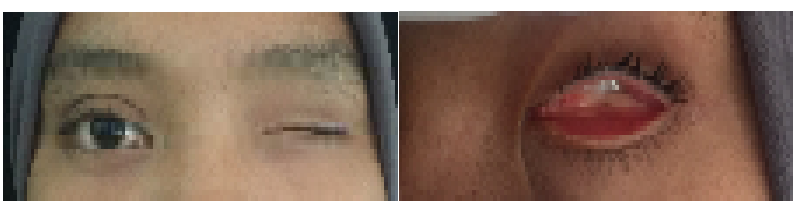

Figure 1 Enucleated left eye

\section{MANAGEMENT}

First, glycerine application was done onto the eyelashes and the lining of anopthalmic socket. A custom tray was fabricated with autopolymerizing polymethyl methacrylate. The tray was finished, polished, and tried in the patient to check for extensions. An impression of the anopthalmic socket was made. Cast was made from type II gypsum on which a special tray was fabricated using self-cure acrylic. A syringe was attached to the special tray through a perforation made at the centre of it. Impression of the defect was recorded using polyvinyl siloxane light viscosity material. The material was injected into the socket, while the patient was instructed to make various eye movements as the material was injected so that the impression was recorded in the functional form. After the material had set, impression was retrieved from the socket and checked to ensure that all the surfaces were recorded. A two-piece type IV dental stone cast was poured to immerse the lower part of the impression (Fig.2). After the stone had set, separating media was applied on the surface. Then a second layer was poured. Marking was made on all the four sides of cast for proper reorientation of the cast. Next, the wax pattern was fabricated by pouring the molten wax into the impression. The wax was properly contoured and carved to give it a simulation of the lost eye. The wax pattern was tried in patient's socket and checked for size, comfort, support, fullness, and retention by performing the functional movements. Iris positioning was performed using face symetris measurement tool.

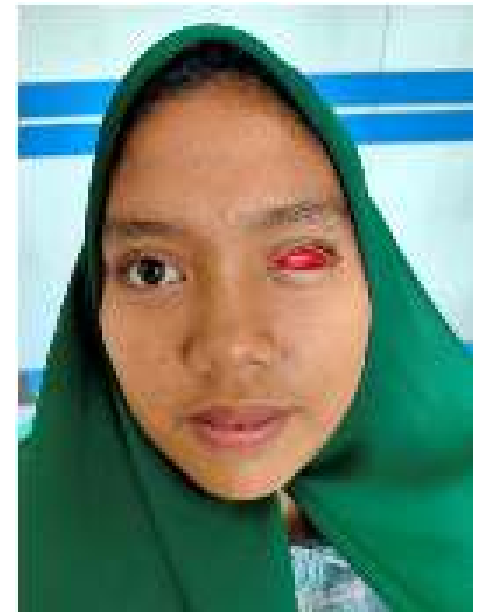

Figure 2 Wax Sclera Try-in

Iris fabrication was done using mobile photography. The photo was taken in a dark room, a white flashlight positioned $45^{\circ}$ from the patienteye was used as light source. The smart phone with built in macro features was positioned in front of the patient eye (Fig.3).

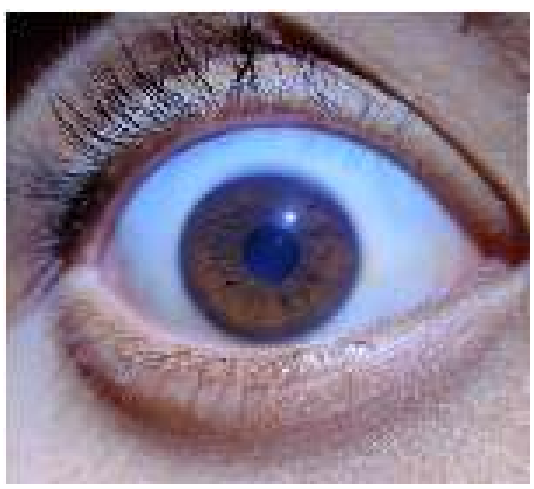

Figure 3 Mobile photography result

The photo was edited with photo editing software (Adobe Photoshop CC 2015) to eliminate the white flash light reflection from the eye, using the same software (Fig.4), a custom ocular shade guide was made with a saturation and lightness gradation to compensate the biased effect from the light source. The custom ocular shade guide was printed and ready to use (Fig.5).

After the iris matching was done, the selected iris was cut with a curved scissors and coated with cyanoacrylate adhesive. The iris button was fabricated with a custom cuvette. A layer of self-cured transparant polymethyl-methacrylate was placed on the custom cuvette, then the iris photo was attached on top of it with an adhesive. A hot cured transparant polymethyl-metacrylate was placed on top of the iris photo. and the second part of the custom cuvettewas placed and both of it was pres- 
sed with a press machine. The excess material was removed and the custom cuvette was put into a hot water. After the hot cure polymethyl-methacrylate sets, the custom cuvette was opened and the iris button was polished.

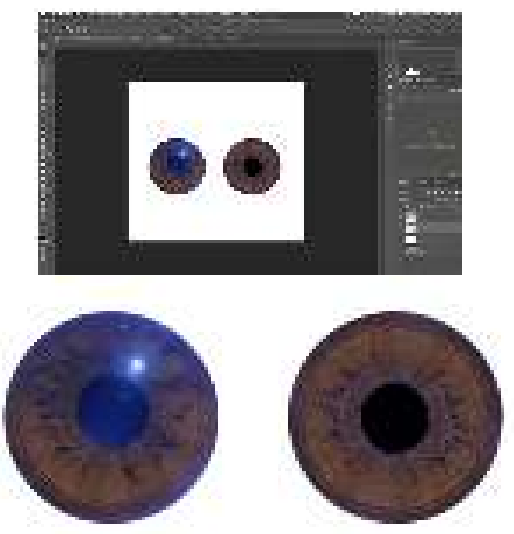

Figure 4 Photo editing result

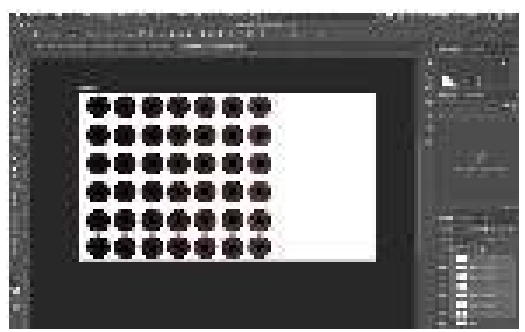

Figure 5 Custom ocular shade guide

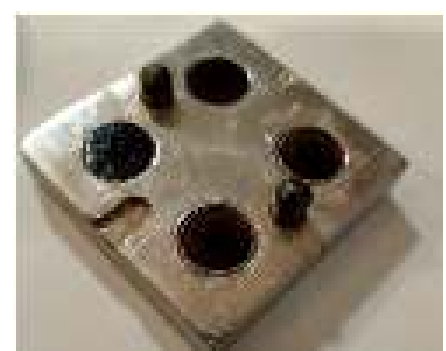

Figure 6 Iris button fabrication.

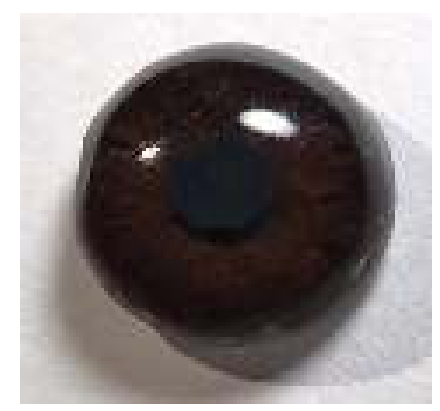

Figure 7 Iris button

After the iris button fabrication (Fig.6) process completed, the iris button (Fig.7) was attached into the wax sclera and the final try-in was done in the patient (Fig.8). The wax sclera with iris button at- tached was invested in a cuvette (Fig.9). Dewaxing was done and continued with sclera fabrication using hot cured transparent polymethyl-methacrylate mixed with acrylic paint matching the patient sclera color.

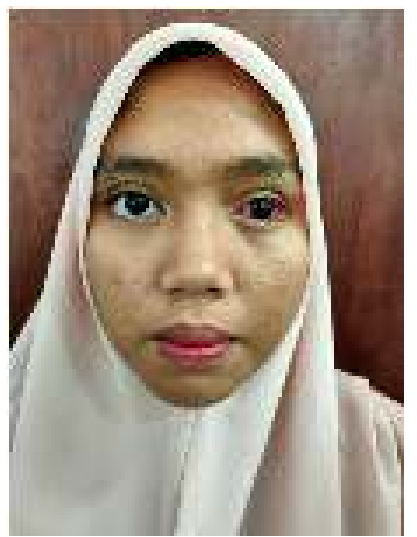

Figure 8 Final try-in

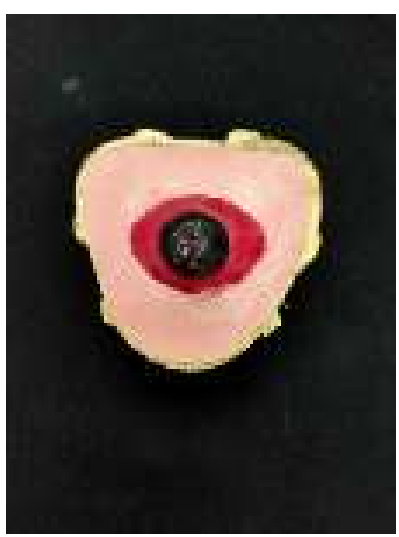

Figure 9 Wax sclera

After deflasking, a $2 \mathrm{~mm}$ reduction with stone bur was done on the surface of the ocular prosthesis, characterization was done using a color penciland a red wool filament mimicking the patient eye. The prosthesis was placed into the cuvette and a transparent hot cured polymethyl-methacrylate was placed on top of it. After the curing process completed the ocular prosthesis was deflasked and polished (Fig.10). The ocular prosthesis was inserted into the patient socket (Fig.11) and the patient was instructed to return for control.

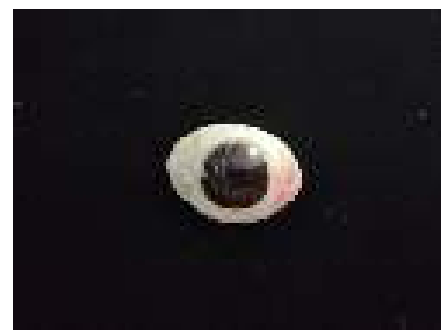

Figure 10 Final ocular prosthesis 


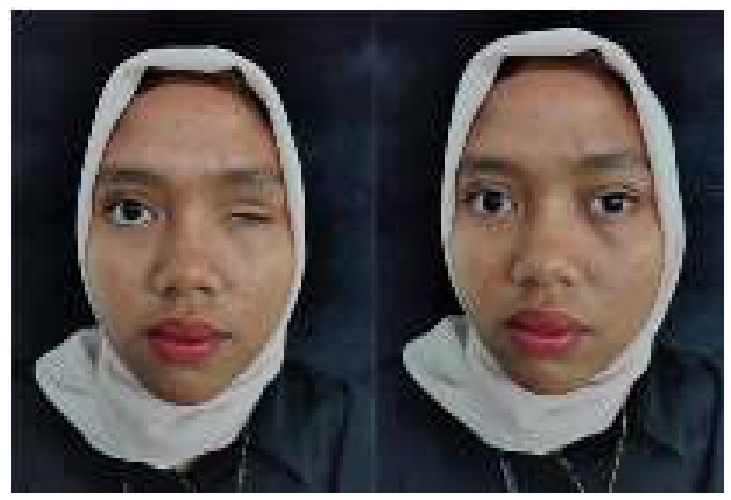

Figure 11 Final result

\section{DISCUSSION}

Custom ocular prosthesis is the best treatmentoption in prosthetic eye replacement. It provides better adaptation, esthetic, and eye movement. It also preserves the remaining tissue post enucleation. ${ }^{1,2}$ When it comes to the ocular prosthesis esthetics, many clinicians have concluded that iris color is the most important consideration. Thecommon techniques for the fabrication of custom-made prosthesis are paperiris disk and blank iris disk technique. However, painting the iris disk involves both artistic skills and science of color. This method of iris shade matching is also time consuming, because the time ittakes for the paint to dry completely. One of the alternatives to overcome the disadvantages of the conventional painting technique is digital photography. ${ }^{6}$

Nowadays, digital photography has evolved, so has colour calibration technology and inkquality. The ability to printan exact colourreplica of what was taken in camera has become much simpler. Digital photography arguably provides a more scientifically robustmethod of production that can be standardised, measured, and effectively reproduced. The disadvantages of digital photographyare the requirement of expertise in photography and the cost of the photography equipment. ${ }^{7}$

Mobilephotography using smartphones have comea long way in digital photography due to the fact that those cameras have some interesting features and characteristics that are beneficial, such as 1) very small aperture due to the small size of the camera and its diaphragm. Therefore, a very high depth of field is achievedon a regularbasis;2) good ISO settings adding more light sensitivity to the camera with low noise; rather good resolution to show small details; 3) large display to preview and view the images, especially when using smart phones with large screens; 4) battery autonomy that allows working for many hours; 5) the white balance, exposure, focus, ISO, metering and the shutter speed can be modified manually in some cameras; 6) smart phones are light and easy to operate. ${ }^{4}$

This benefits, combine with the right technique will allow us to reproduce the patient iris esthetically in a short amount of time. Mobile photography also eliminates the needs for operator skills and experience in painting and mixing color which is required in the conventional methods. ${ }^{8-10}$

The usage of custom occular shade guide will compensate the color biased that might happen during the photo taking or editing process, therefore increasing the precision of the iris color during iris shade matching. Even though this technique provides us with many advantages, it requires the operator skills in using the photo editing software such as adobe photoshop to reproduceanatural and highly esthetic iris.

It is concluded that the novel approach using the mobile photography and custom ocular shade guide simplified the iris shade matching, provide highly esthetic results, eliminate the needs for operator painting skills, and save significantamount of time in fabrication.

\section{REFERENCES}

1. Shah RM, Coutinho I, Chitre V, Aras MJ. The ocular prosthesis: a novel technique using digital photography. J Indian Prosthodont Soc 2014; 14(Suppl 1): 248-54.

2. Lanzara R, Thakur A, Vicwambaran M, Khattak A. Fabrication of ocular prothesis with digital costumization technique. J Family Med Primary Care 2019; 8(3): 1239-42.

3. Gunaseelaraj R. Custom-made ocular prosthesis. J Pharm Bioallied Sci 2012; 4(Suppl 2): s177-9.

4. Hardan LS. Mobile dental photpgraphy: a simple technique for documentation and communication. Quintessence Int 2020;51(6):510-8.

5. Aggarwal H, Singh RD, Kumar P, Gupta SK, Alvi HA. Prosthetic guidelines for ocular rehabilitation in patients with phthisis bulbi: A treatment-based classification system. J Prosthet Dent 2014;111(6):525-8.

6. Ahmad N, Verma AK, Ali M, Srivastava M. Prosthetic rehabilitation of an ocular defect with customized iris: a case report. Malaysian Dent J 2014;36(2):1-5.

7. Zoltie T, Walshaw EG. Digital photographic technique for the production of an artificial eye. J Visual Communication in Med 2021;44(2):41-4.

8. Buzayan MM. Ocular defectrehabilitation using photography and digital imaging: a clinical report. J Prosthodont 
60 Joseph Ginting, et al: Novel approach in iris shade matching: mobile photography and custom ocular

2015; 24: 506-10.

9. Jain S, Makkar S, Gupta S, Bhargava A. Prosthetic rehabilitation of ocular defect using digital photography: a case report. J Indian Prosthodont Soc 2010; 10(3): 190-3.

10. Chamaria A, Aras M, Chitre V, Bhushan P.Ocular defect rehabilitation using digital photography. Int J Orofac Res 2017;2(1):32-4. 